\title{
The Revised Telephonic Triage of New Jersey and Conventional Method of Triage by Emergency Medical Dispatchers of Qom Emergency Medical Services, Iran, in 2012: A Comparative Investigation
}

\author{
Mohammad Parvaresh Masoud, ${ }^{1,}{ }^{*}$ Mahryar Yoldashkhan, ${ }^{2}$ Sima Zohari Anboohi, ${ }^{3}$ Nezhat Shakeri, ${ }^{4}$ \\ Mahdi Kashani Nejad, ${ }^{5}$ Mohammad Javad Bagheri, ${ }^{5}$ Mohsen Chavoshi, ${ }^{5}$ and Hamid Asayesh ${ }^{1}$ \\ ${ }^{1}$ School of Paramedical Sciences, Qom University of Medical Sciences, Qom, IR Iran \\ ${ }^{2}$ Department of Anesthesia and Operating Room, School of Nursing and Midwifery, Shahid Beheshti University of Medical Sciences, Tehran, IR Iran \\ ${ }^{3}$ Department of Nursing, School of Nursing and Midwifery, Shahid Beheshti University of Medical Sciences, Tehran, IR Iran \\ ${ }^{4}$ Department of Biostatistics, School of Paramedical Sciences, Shahid Beheshti University of Medical Sciences, Tehran, IR Iran \\ ${ }^{5}$ Emergency Medical Center, Qom University of Medical Sciences, Qom, IR Iran \\ "Corresponding author: Mohammad Parvaresh Masoud, School of Paramedical Sciences, Qom University of Medical Sciences, Qom, IR Iran. Tel: +98-9122513361, Fax: \\ +98-25333209123, E-mail: m_parvaresh_m@yahoo.com
}

Received 2016 July 13; Accepted 2016 August 13.

\begin{abstract}
Background: The demand for medical emergencies often begins with a call to a dispatch center. Appropriate performance of this unit significantly influences providing effective services.

Objectives: The current study aimed to compare the effect of using telephone triage manual revised by New Jersey and the conventional methods on accuracy of triage done by 115 emergency medical dispatchers of Qom, Iran, in 2012.

Methods: This quasi-experimental study aimed to compare the effects of two conventional and New Jersey revised methods on performance of emergency medical dispatchers of Qom. Conventional (September and October) and New Jersey data (December and January) were extracted and compared. November was skipped due to emergency medical dispatcher familiarity with the program. Results: Performing the new method non-significantly changed the response time $(\mathrm{P}=0.053)$ and communication skill $(\mathrm{P}=0.053)$. Scientific and medical skills $(\mathrm{P}=0.000)$ were significantly changed. Overall performance significantly improved $(\mathrm{P}=0.000)$.

Conclusions: The revised New Jersey method significantly increased appropriate overall performance of the communication experts and decreased their dysfunction.
\end{abstract}

Keywords: Emergency Medical Service, Emergency Medical Dispatch and Communication, Telephone Triage, Emergency Medical Dispatchers

\section{Background}

Emergency medical service (EMS) is a comprehensive system, which makes an effective relationship between staff, equipment and facilities. EMS aims to provide effective medical and immunity services, which should be presented coordinately and timely to the victims of the diseases and events (1). Medical emergencies are the first cause of contact by the majority of people with the health systems in emergency and life-threatening cases; it is as the goalkeeper of accessing to the secondary and tertiary services. This system is enjoying new and advanced technologies to take care of the patients and injuries (2).

Chain of pre-hospital cares is started by calling the emergency medical dispatch (EMD); a situation, which is associated with different degrees of stress and anxiety of the caller; some conditions, which can distort receiving accurate and complete information $(3,4)$. Calls will be prioritized by EMD based on medical information and other health related information. Operator offers some advice to the caller and informs the ambulance staff regarding the situation at the scene at the same time (5). Basic information about the mission, obtained from the dispatch unit, is counted as the starting point of pre-hospital cares (6).

Dispatch unit makes the caller sure of receiving the required medical care (7). The emergency operator can strengthen sense of security and trust in patients; while he/she is waiting for the ambulance, this sense is built and strengthened through telephone contact $(3,8,9)$. However, 
there are some problems with the diagnosis of medical problems of the callers through telephone contact, since the patient cannot be observed. Some studies are done in this regard, indicating that operators did not diagnose serious medical problems. One of the aims of medical emergency systems is making a balance between available resources to provide for the patients and the amount of patients' need to them (10-12).

Considering 1000 - 1200 calls per 24 hours to the communication and dispatch unit of Qom Emergency medical service and circadian 140 - 160 missions and limited number of ambulance, dealing properly with each call and appropriate telephone triage is vital. Totally, there are 32 emergency bases in Qom, which are divided into two 16 urbane bases and 16 road bases. Considering these conditions and a population of about one million and twohundred thousand in Qom and also considering that the population increases on some special days and occasions because of pilgrims, inappropriate triage increases missions, response time to the real patients, staff's fatigue and power reduction and results in depreciation of hardware equipment (medical equipment, ambulance) and increased complaints.

\section{Objectives}

The current study aimed to compare the use of revised telephone triage manual of New Jersey and the conventional methods in terms of accuracy of triage by 115 emergency medical dispatchers of Qom in 2012.

\section{Methods}

This quasi-experimental study was conducted in 2012; there were two study populations including calls and medical dispatchers (female nurses who were answering the calls in 115 emergency unit). Samples of the study (medical dispatchers) were the study population. By call it is meant all the calls to 115 emergency of Qom in 2012 regarding medical emergency services demand, answered by medical dispatchers. Among 200 assessed calls by the quality control unit in September and October 2012, 150 cases were randomly selected by conventional method. After performing New Jersey revised method, November was skipped due to emergency medical dispatcher familiarity with the new method and among 230 assessed calls by quality control unit in December and January, 150 cases were randomly selected.

New Jersey telephone triage instruction of 2009 edition was translated. Considering their pre-hospital emergency system differences with Iran, the cases related to ba- sic life support (BLS) and advanced life support (ALS) systems separation were integrated. Finally a set was prepared consisting of a general section including a list of all cases and frequently asked questions (FAQ) on every call, which assessed general profile of the callers and lifethreatening issues such as coma and items related to airway, breathing and circulation. The final list included trauma emergencies (red), medical emergencies (blue), critical and life-threatening events (green) and others (orange). Medical dispatchers asked the common questions and found one of the diseases, then clicked on the related part and the related page was opened, which included three parts: specific questions, dispatch cases and guides before dispatch. In any part, if cardiopulmonary resuscitation (CPR) was required, the medical dispatcher clicked on the CPR part and found the instructions based on the age group (infant, children and adult) and guided the caller according to the latest CPR instructions of American heart association (2010). Then this set was given to faculty members, technical and training experts, center doctors, head of quality control, assistance and head of Qom Emergency medical service to be studied by them. After obtaining their comments, necessary changes were made to design a usable set for Qom emergency communication unit, which can be performed in the city. Related contents and images were developed and prepared by different softwares such as Photoshop etc.; then, the output was saved on executable files to be used through computer systems in the center.

To be more familiar with the staff of communication unit, all the 12 females, who had Bachelor of Science in nursing, were invited to participate in a class, held for about four hours and the set was reviewed by them. Formal missions were defined in this review and new instructions were used for medical dispatchers' answering to these missions. In the next step, the prepared program by information technology (IT) unit was installed in the computers and the method of working with a new program was reviewed for about one month by the presence of a researcher in the communication unit to make sufficient proficiency in medical dispatchers; it was confirmed by the head of communication and dispatch unit, technical expert and head of quality control. Then all the medical dispatchers used new instructions and their results were assessed.

Then the researcher referred to the quality control unit, introduced himself and explained the aim of the study, asked the manager to listen to the conversations and record the results before performing New Jersey revised telephonic triage and then give the forms and the related tables to the researcher. For the cases after performing new instructions, prepared forms and tables were used in 
September and January.

\section{Results}

According to Table 1, all the staff of communication unit was female and had bachelor of science in nursing, 118 (78.66\%) missions were obtained through conventional method during the standard time and 32 (21.33\%) missions were obtained beyond the standard time and these cases through New Jersey method were respectively 111(74\%) and $39(26 \%)$. The obtained results were not significant through Chi-square test and 99\% confidence intervals (CI) (P = 0.342 ). It is necessary to mention that in terms of quality control, the standard time to obtain the mission is three minutes and less.

According to Table 2, medical dispatchers' communication skills, including two components, how to answer and master the mental skills, which were compared in the two conventional and New Jersey modified methods. Answering in the conventional method was performed properly in $58.66 \%$ of the cases, which increased to $72 \%$ through performing New Jersey method; improper answering decreased from $33.33 \%$ to $22.66 \%$; offensive answering also decreased from $8 \%$ to $5.33 \%$. These results were not significant through Chi-square test and 99\% CI $(\mathrm{P}=0.053)$. The changes were exactly the same in terms of mastery of mental skills.

According to Table 2, scientific and medical skills of the medical dispatchers, including the two components of history taking and scientific proficiency were compared in the two revised New Jersey and conventional methods. According to Table 3, overall improvement was observed in history taking through New Jersey method. Therefore, the good case in conventional method was $23.33 \%$, which increased to $47.33 \%$ through New Jersey method. The moderate case decreased from 52\% to 39.33\% through New Jersey method. Finally the weak case from $24.66 \%$ in conventional method decreased to $13.33 \%$ through New Jersey method. The changes were significant through Chi-square test and 99\% CI $(\mathrm{P}=0.000)$. The changes were exactly the same in terms of scientific proficiency.

According to Table 4, total performance, which might be the most important component of evaluating a medical dispatcher, based on the quality control unit approach, had a significant improvement, which was significant through Chi-square test and 99\%CI $(\mathrm{P}=0.000)$. Therefore, appropriate performance in conventional method 59.33\% improved to $79.33 \%$ through New Jersey method and inappropriate performance in the conventional method $40.66 \%$ decreased to $20.66 \%$ through New Jersey method.

\section{Discussion}

All the staff of emergency unit of Qom was female and had BA in nursing. In different countries with different ethnics and races in dispatch units answer telephones and are provide medical services. Several studies defended the presence of nurses in these units and considered it as a useful method $(9,13,14)$

Quality control unit experts consider three topics to score total performance of medical dispatchers: 1) duration of obtaining the mission, 2) medical dispatchers' communication skills, including how to answer and master the mental skills and 3) medical dispatchers' scientific and medical skills, including how to take history and scientific proficiency.

There are different issues regarding the effect of prioritization system (triage instruction) on mission time. Critics believe that such questions result in delay in mission, but proponents of this system believe that such questions require little time and result in appropriate, safe and effective answer. It is believed that only $5 \%-10 \%$ of emergency missions are critical and life-threatening and there is enough time to communicate with the patients in other cases (15). Considering these approaches and results of the final performance, it seems that a little increase in time of obtaining mission does not interfere in the process of helping patients. The present study also showed that the longest time of obtaining mission was about 3 minutes and 30 seconds, but the total results, which led to increased total performance was really significant.

Results of medical dispatchers' communication skills, including how to answer and mental mastery indicated that the changes were not significant; however, there was a narrow difference with the significant level of 0.003; the main cause was people and callers' behavior; no work in this regard was done in this study and no change was made and unfortunately some callers insulted the operators. They do not let to complete the performance of the instructions; therefore, more education is required. Lack of criminal law dealing with callers who have offensive behavior is another cause of this problem, since it makes stress and anxiety in medical dispatchers and sometimes they show reaction to such behaviors.

There are two problems in communication and dispatch unit regarding anxious callers and people familiar with medical issues such as nurses and doctors. The first group does not provide adequate and correct information because of their anxiety and does not follow the instructions before an ambulance arrives. The second group expects rapid ambulance dispatch because of their medical information and does not provide adequate information to the operators. Working under medical emergency 
Table 1. Comparison of Duration of Mission Taking in Communication Unit of Qom Emergency Medical Service in the Two Conventional and New Jersey Methods in 2012 ${ }^{\mathrm{a}}$

\begin{tabular}{lccc}
\hline \multirow{2}{*}{ Duration of Mission Taking } & \multicolumn{2}{c}{ Common } & New Jersey \\
\cline { 2 - 4 } & Frequency & Percent & Frequency \\
\hline To the standard extent & 118 & 78.66 & 111 \\
beyond the standard extent & 32 & 21.33 & 39 \\
Total & 150 & 100 & 150 \\
& & Chi-square $=0.904, \mathrm{df}=1, \mathrm{P}=0.342$ & 100 \\
\hline
\end{tabular}

${ }^{a}$ Duration of mission taking in two conventional and New Jersey revised methods was compared. According to the quality control unit, the standard time to take the mission was up to three minutes and less.

Table 2. Comparison of Communication Skills in Communication Unit of Qom Emergency Medical Service in Two Conventional and New Jersey Methods in $2012^{\mathrm{a}}$

\begin{tabular}{|c|c|c|c|c|c|c|c|}
\hline \multirow{3}{*}{$\begin{array}{l}\text { The method of answering } \\
\text { Method }\end{array}$} & \multicolumn{6}{|c|}{ Communication Skills } & \multirow[t]{3}{*}{ P Value } \\
\hline & \multicolumn{2}{|c|}{ Appropriate, No. (\%) } & \multicolumn{2}{|c|}{ Inappropriate, No. (\%) } & \multicolumn{2}{|c|}{ Offensive, No. (\%) } & \\
\hline & Conventional & NewJersey & Conventional & New Jersey & Conventiona & New Jersey & \\
\hline Results & $88(58.66)$ & $108(72)$ & $50(33.33)$ & $34(22.66)$ & $12(8)$ & $8(5.33)$ & 0.053 \\
\hline Mastery of mental skills & \multicolumn{2}{|c|}{ Good, No. (\%) } & \multicolumn{2}{|c|}{ Moderate, No. (\%) } & \multicolumn{2}{|c|}{ Weak, No. (\%) } & \\
\hline Method & Conventional & New Jersey & Conventional & New Jersey & Conventional & New Jersey & \\
\hline Results & $88(58.66)$ & $108(72)$ & $50(33.33)$ & $34(22.66)$ & $12(8)$ & $8(5.33)$ & 0.053 \\
\hline
\end{tabular}

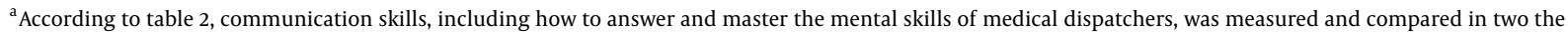
conventional and New Jersey revised methods in 2012.

Table 3. Comparison of Scientific and Medical Skills in Communication Unit of Qom Emergency Medical Service in Two Conventional and New Jersey Methods in $2013^{\mathrm{a}}$

\begin{tabular}{|c|c|c|c|c|c|c|c|}
\hline \multirow{3}{*}{$\begin{array}{l}\text { How to take history } \\
\text { Method }\end{array}$} & \multicolumn{6}{|c|}{ Medical and Scientific Skill } & \multirow[t]{3}{*}{ PValue } \\
\hline & \multicolumn{2}{|c|}{ Appropriate, No. (\%) } & \multicolumn{2}{|c|}{ Inappropriate, No. (\%) } & \multicolumn{2}{|c|}{ Offensive, No. (\%) } & \\
\hline & Conventional & New Jersey & Conventional & New Jersey & Conventional & New Jersey & \\
\hline Results & $35(23.33)$ & $71(47.33)$ & $78(52)$ & $59(39.33)$ & $37(24.66)$ & $20(13.33)$ & 0.000 \\
\hline Scientific proficiency & \multicolumn{2}{|c|}{ Good, No. (\%) } & \multicolumn{2}{|c|}{ Moderate, No. (\%) } & \multicolumn{2}{|c|}{ Weak, No. (\%) } & \\
\hline Method & Conventional & New Jersey & Conventional & New Jersey & Conventional & New Jersey & \\
\hline Results & $35(23.33 \%)$ & $71(47.33)$ & $78(52 \%)$ & $59(39.33)$ & $37(24.66 \%)$ & $20(13.33)$ & 0.000 \\
\hline
\end{tabular}

${ }^{a}$ Scientific and medical skills, consisted of how to take history and scientific proficiency were compared and measured in the two conventional and New Jersey revised methods.

conditions whether on the scene or behind the scene are specified with some features such as working under pressure, being multitasking, making effective and rapid decisions, working in shifts of 24 hours a day and seven days a week and managing different emotional responses. The role of dispatch unit staff is more sensitive because they are counted as the entry point of pre-hospital emergency. Therefore, operators need to collect a lot of information and extract the related ones to provide them to the appropriate units (16).

Results achieved from medical dispatchers' scientific and medical skills, including the components of history taking and scientific proficiency, indicated that the applied changes were significant. There was no independent case as the scientific proficiency in the studies because some believe that protocol and acting accordingly increases the operator's detection power. On the other hand, some researchers indicated direct relationship of protocol with increased medical knowledge of the triage nurse $(17,18)$. According to the mentioned components, it was observed that performing New Jersey revised protocol significantly improved total performance of medical dispatchers.

Kuisma et al. showed that the presence of a functional dispatch unit is effective to identify high-risk patients who 
Table 4. Comparison of Total Performance in Communication Unit of Qom Emergency Medical Service in Two Conventional and New Jersey Methods in $2012^{\mathrm{a}}$

\begin{tabular}{lcccc}
\hline \multirow{2}{*}{ Total Performance } & \multicolumn{2}{c}{ Conventional } & \multicolumn{2}{c}{ New Jersey } \\
\cline { 2 - 5 } & Frequency & Percent & Frequency & Percent \\
\hline Appropriate & 89 & 59.33 & 119 & 79.33 \\
Inappropriate & 61 & 40.66 & 31 & 20.66 \\
Total & 150 & 100 & 150 & 100 \\
& Chi-square $=14 / 110, \mathrm{df}=1, \mathrm{P}=0 / 000$ & \\
\hline
\end{tabular}

${ }^{\mathrm{a}}$ Total performance in the two conventional and New Jersey revised methods were measured. This case included two types of appropriate and inappropriate total performances.

need emergency cares (19). Some studies disagree; for example, Maatta et al. in Finland stated that dispatch unit reforms did not play any roles in correct assessment of patients with life-threatening problems. One of the essential tools for appropriate performance of communication unit staff is a systematic and scientific instruction to make appropriate decisions accordingly in moments of crisis. Without protocols, decisions are based on the personal interpretations. The possibility of error is common in such a system, since communication unit is a non-visual, vague and complicated unit and also it is in emergency conditions. Quality control measures are not standard in such conditions because there is no unique instruction to measure its outcomes; therefore, the quality control unit is not going to detect the problems and errors until they occur (20).

One of the causes of differences between the studies is the evaluation criteria. Dispatch unit plays an important and basic role in providing emergency services to the patients and there are some problems in evaluating its quality and effect on medical emergencies (1).

Applying the present study in three educational, clinical and management areas is recommended as follows. It is recommended to design educational resources by considering the curriculum content and the importance of dispatch unit to conduct effective relationships between people and medical emergency systems to be familiar with the role and the importance of dispatch unit in a better way. Second, it is recommended to perform a systematic, scientific and tested protocol similar to those of other dispatch centers of the country.

\subsection{Conclusion}

Totally, performing New Jersey method increased appropriate total performance of medical dispatchers and decreased their appropriate performance significantly. Results of the current study showed that performing New
Jersey method led to low increase of mission taking time and this increase finally led to improvement of the process. Also, performing this instruction improves the method of appropriate history taking, increases scientific proficiency and finally promotes appropriate total performance of medical dispatchers significantly. The method of answering and mastery of the mental skills also improved, not significantly but there was a narrow distance with the significant level (0.003) and the main cause was the callers who did not answer medical dispatchers appropriately, which needs public education.

\section{Acknowledgments}

The researchers thank and appreciate friendly cooperation of quality control and communication unit experts of Qom Emergency medical service research vice chancellor of Shahid Beheshti University (Tehran, Iran) and Qom University of Medical Sciences, Qom, Iran.

\section{References}

1. Moore L. Measuring quality and effectiveness of prehospital EMS. Prehosp Emerg Care. 1999;3(4):325-31. [PubMed: 10534034].

2. Roudsari BS, Nathens AB, Cameron P, Civil I, Gruen RL, Koepsell TD, et al. International comparison of prehospital trauma care systems. Injury. 2007;38(9):993-1000. doi:10.1016/j.injury.2007.03.028. [PubMed: 17640641].

3. Bang A, Ortgren PO, Herlitz J, Wahrborg P. Dispatcher-assisted telephone CPR: a qualitative study exploring how dispatchers perceive their experiences. Resuscit. 2002;53(2):135-51. doi: 10.1016/S03009572(01)00508-1.

4. Forslund K, Quell R, Sorlie V. Acute chest pain emergencies - spouses' prehospital experiences. Int Emerg Nurs. 2008;16(4):233-40. doi: 10.1016/j.ienj.2008.07.001. [PubMed: 18929341].

5. Crowther L, Williams R. Nurse interventions in ambulance command-and-control centres. Emerg Nurse. 2009;17(8):22-5. doi: 10.7748/en2009.12.17.8.22.c7430. [PubMed: 20043428].

6. Hjalte L, Suserud BO, Herlitz J, Karlberg I. Why are people without medical needs transported by ambulance? A study of indications for pre-hospital care. Eur J Emerg Med. 2007;14(3):151-6. doi: 10.1097/MEJ.ob013e3280146508. [PubMed: 17473609].

7. Studnek JR, Thestrup L, Blackwell T, Bagwell B. Utilization of prehospital dispatch protocols to identify low-acuity patients. Prehosp Emerg Care. 2012;16(2):204-9. doi: 10.3109/10903127.2011.640415. [PubMed: 22239124].

8. O'Cathain A, Turner J, Nicholl JP. The acceptability of an emergency medical dispatch system to people who call 999 to request an ambulance. Emerg Med J. 2002;19(2):160-3. [PubMed: 11904272].

9. Forslund K, Kihlgren M, Ostman I, Sorlie V. Patients with acute chest pain - experiences of emergency calls and pre-hospital care.J Telemed Telecare. 2005;11(7):361-7. doi: 10.1258/135763305774472006. [PubMed: 16238838].

10. Beillon LM, Suserud BO, Karlberg I, Herlitz J. Does ambulance use differ between geographic areas? A survey of ambulance use in sparsely and densely populated areas. Am J Emerg Med. 2009;27(2):202-11. doi: 10.1016/j.ajem.2008.01.012. [PubMed: 19371529].

11. Calle P, Vanhaute O, Lagaert L, Houbrechts H, Buylaert W. The 'early access' link in the chain of survival for cardiac arrest victims in Ghent, Belgium. Eur J Emerg Med. 1994;1(3):145-8. [PubMed: 9422158]. 
12. Buck BH, Starkman S, Eckstein M, Kidwell CS, Haines J, Huang R, et al. Dispatcher recognition of stroke using the National Academy Medical Priority Dispatch System. Stroke. 2009;40(6):2027-30. doi: 10.1161/STROKEAHA.108.545574. [PubMed: 19390065].

13. Dale J, Higgins J, Williams S, Foster T, Snooks H, Crouch R, et al. Computer assisted assessment and advice for "non-serious" 999 ambulance service callers: the potential impact on ambulance despatch. Emerg Med J. 2003;20(2):178-83. [PubMed: 12642540].

14. Griffiths K. Emergency medical dispatch: no longer the 'weak link'? JEMS. 2003;28(10):52-8. [PubMed:14583703].

15. Farand L, Leprohon J, Kalina M, Champagne F, Contandriopoulos AP, Preker A. The role of protocols and professional judgement in emergency medical dispatching. Eur J Emerg Med. 1995;2(3):136-48. [PubMed: 9422199].

16. Clawson JJ, Sinclair R. The emotional content and cooperation score in emergency medical dispatching. Prehosp Emerg Care. 2001;5(1):29-
35. [PubMed: 11194066].

17. Wilson S, Cooke M, Morrell R, Bridge P, Allan T. A systematic review of the evidence supporting the use of priority dispatch of emergency ambulances. Prehosp Emerg Care. 2002;6(1):42-9. [PubMed: 11789649].

18. Wahlberg AC, Cedersund E, Wredling R. Bases for assessments made by telephone advice nurses. J Telemedicine Telecare. 2005;11(8):403-7. doi: 10.1258/135763305775013581.

19. Kuisma M, Boyd J, Vayrynen T, Repo J, Nousila-Wiik M, Holmstrom P. Emergency call processing and survival from out-of hospital ventricular fibrillation. Resuscitation. 2005;67(1):89-93. doi: 10.1016/j.resuscitation.2005.04.008. [PubMed: 16129542].

20. Maatta T, Kuisma M, Vayrynen T, Nousila-Wiik M, Porthan K, Boyd J, et al. Fusion of dispatching centres into one entity: effects on performance. Acta Anaesthesiol Scand. 2010;54(6):689-95. doi: 10.1111/j.13996576.2010.02243.x. [PubMed: 20455880]. 